\title{
Exploring planetary magnetic environments using magnetically unclean spacecraft: a systems approach to VEX MAG data analysis
}

\author{
S. A. Pope ${ }^{1}$, T. L. Zhang ${ }^{2,3}$, M. A. Balikhin ${ }^{1}$, M. Delva ${ }^{3}$, L. Hvizdos ${ }^{4}$, K. Kudela ${ }^{4}$, and A. P. Dimmock ${ }^{1}$ \\ ${ }^{1}$ Department of Automatic Control and Systems Engineering, University of Sheffield, Mappin Street, Sheffield, S1 3JD, UK \\ ${ }^{2}$ Department of Geophysics and Planetary Sciences, University of Science and Technology of China, Hefei, 230026, China \\ ${ }^{3}$ Space Research Institute, Austrian Academy of Sciences, Schmiedlstrasse 6, 8042 Graz, Austria \\ ${ }^{4}$ Institute of Experimental Physics, Slovak Academy of Sciences, Watsonova 47, Kosice, Slovakia
}

Received: 27 January 2011 - Revised: 25 March 2011 - Accepted: 28 March 2011 - Published: 8 April 2011

\begin{abstract}
In situ measurements of the magnetic field are vital to the study of many fundamental problems in planetary research. Therefore the magnetometer experiment is a key element of the payload of Venus Express. In addition to the interaction of the solar wind with Venus, these measurements are crucial for the study of atmospheric escape and detection of lightning. However, the methodology for the magnetic field measurements had to be different to the traditional approach, because Venus Express is not a magnetically clean spacecraft. A technique based on two-point simultaneous measurements of the magnetic field and systems identification software is used to separate the natural magnetic field from the spacecraft generated interference. In this paper an overview of the techniques developed to separate these two field types and the results achieved for $1 \mathrm{~Hz}$ Venus Express data are presented. Previous publications suggest that the resulting Venus Express cleaned data is of comparable quality to measurements made from onboard magnetically clean spacecraft (Zhang et al., 2008a, b; Slavin et al., 2009).
\end{abstract}

Keywords. Magnetospheric physics (Solar wind interactions with unmagnetized bodies) - General or miscellaneous (Instruments useful in three or more fields; Techniques applicable in three or more fields)

\section{Introduction}

The scientific payload of Venus Express (VEX) includes MAG, which is an experiment to make in situ measurements of the local magnetic field vector. These measurements are

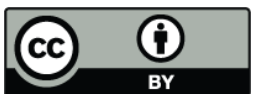

Correspondence to: T. L. Zhang

(tlzhang@ustc.edu.cn) crucial to studying the interaction of the solar wind with Venus (Zhang et al., 2007). However, the VEX spacecraft bus is a twin to that of Mars Express, which was not designed to be magnetically clean or to carry a magnetometer. As a consequence there are severe implications for the data processing procedures applied to the measurements made by MAG. This is because the local natural field is superimposed onto the spacecraft generated magnetic field vector, such that the field measured by the MAG sensor can be described by Eq. (1).

$\boldsymbol{B}_{\mathrm{M}}(t)=\boldsymbol{B}_{\mathrm{N}}(t)+\boldsymbol{B}_{\mathrm{S}}(t)+\boldsymbol{B}_{\mathrm{L}}(t)+\boldsymbol{B}_{\mathrm{I}}(t)$.

In Eq. (1), indices $\mathrm{M}, \mathrm{N}, \mathrm{S}, \mathrm{L}$ and $\mathrm{I}$ are used to denote the measured field, natural field, spacecraft generated field, magnetometer zero level offset and instrument noise, respectively. Equation (2) describes the further decomposition of the spacecraft generated field into dynamic $\boldsymbol{B}_{\mathrm{Sd}}$ and static $\boldsymbol{B}_{\mathrm{Ss}}$ components.

$\boldsymbol{B}_{\mathrm{S}}(t)=\boldsymbol{B}_{\mathrm{Sd}}(t)+\boldsymbol{B}_{\mathrm{Ss}}$

To attain an accurate measure of the natural magnetic field, the contributions of $\boldsymbol{B}_{\mathrm{L}}, \boldsymbol{B}_{\mathrm{Sd}}$ and $\boldsymbol{B}_{\mathrm{Ss}}$ to the measured field need to be identified. The basic methodology of the Venus Express data cleaning has been described briefly by Zhang et al. $(2008 \mathrm{a}, \mathrm{b})$. The present paper describes how the problem has been formulated, presents the characteristics of the spacecraft magnetic field observed for Venus Express, provides an overview of the details of the identification algorithms developed and presents some of the results attained for the natural magnetic field $\boldsymbol{B}_{\mathrm{N}}$. An in-depth presentation of the details of all of the algorithms is beyond the scope of this paper and these will be presented in subsequent work.

Published by Copernicus Publications on behalf of the European Geosciences Union. 


\section{The MAG experiment and spacecraft generated magnetic field}

The MAG experiment (Zhang et al., 2006) adopts a method where the magnetic field is measured simultaneously at two separate locations. This method is referred to as the Dual Point Method (DPM). DPM was first discussed by Ness et al. (1971) and has already been used for magnetometer experiments carried by spacecraft which have previously explored the Venusian plasma environment, such as Mariner 10 (Ness et al., 1974) and Pioneer Venus Orbiter (PVO) (Snare and Means, 1977). It has recently been used on the Double Star mission (Carr et al., 2005). VEX is the first project in which the DPM is executed for the combined problem of a spacecraft with no substantial magnetic cleaning program and a short magnetometer boom. In the case of VEX the two measurement locations used to implement the DPM are relatively close: $0.1 \mathrm{~m}$ (inboard sensor) and $1 \mathrm{~m}$ (outboard sensor), to the body of the spacecraft. As a comparison, for Double Star the magnetometers were positioned substantially further from the body of the spacecraft $(2.6 \mathrm{~m}$ and $3.1 \mathrm{~m})$. Following magnetic cleaning the only stray-field present in the field vector measured by Double Star were those associated with errors in the layout of the solar power system. In the case of PVO and Mariner 10 the outer sensors were attached $3 \mathrm{~m}$ and $5.8 \mathrm{~m}$ from the respective spacecrafts. This was adequate to sufficiently attenuate any significant spacecraft field at the measurement location. As noted by Ness et al. (1971) "it does not seem prudent to require nor possible to achieve an accurate magnetic map of the spacecraft magnetic field pre-launch in every conceivable operational mode, as demanded by a single magnetometer experiment". This is the case for VEX and excluding several feasibility studies, no analysis or determination of the spacecraft field has been performed prior to launch.

The spatial separation of the two magnetometers is significantly smaller than the characteristic spatial scales of the natural magnetic field near Venus. Therefore it is reasonable to assume that the value of $\boldsymbol{B}_{\mathrm{N}}$ will be the same for both probes, i.e. $\boldsymbol{B}_{\mathrm{Ni}}=\boldsymbol{B}_{\mathrm{No}}=\boldsymbol{B}_{\mathrm{N}}$ (indices "i" and "o" denote measurement made by the inboard and outboard sensors, respectively). Based on this assumption the measurements at the two sensor locations can be described by Eqs. (3) and (4). In such a situation the difference $\boldsymbol{B}_{\mathrm{D}}$ (Eq. 5) between the measurements made by the two sensors is a function of the spacecraft generated field and instrument offsets.

$$
\begin{aligned}
& \boldsymbol{B}_{\mathrm{Mi}}(t)=\boldsymbol{B}_{\mathrm{Ci}}(t)+\boldsymbol{B}_{\mathrm{N}}(t), \\
& \boldsymbol{B}_{\mathrm{Mo}}(t)=\boldsymbol{B}_{\mathrm{Co}}(t)+\boldsymbol{B}_{\mathrm{N}}(t), \\
& \boldsymbol{B}_{\mathrm{D}}(t)=\boldsymbol{B}_{\mathrm{Mi}}(t)-\boldsymbol{B}_{\mathrm{Mo}}(t)=\boldsymbol{B}_{\mathrm{Ci}}(t)-\boldsymbol{B}_{\mathrm{Co}}(t) .
\end{aligned}
$$

In Eqs. (3)-(5) the index $C$ indicates the combined value of the spacecraft field, instrument zero level offset and measurement noise. Importantly $\boldsymbol{B}_{\mathrm{D}}$ does not depend on the natural magnetic field and is only a function of the spacecraft system effects. It contains information on all of the changes in the spacecraft field. This signal is the basis of the techniques developed to identify spacecraft interference in MAG data.

$$
\frac{\left|\boldsymbol{B}_{\mathrm{Mo}}(t+d t)-\boldsymbol{B}_{\mathrm{Mo}}(t)\right|}{\left|\boldsymbol{B}_{\mathrm{Mi}}(t+d t)-\boldsymbol{B}_{\mathrm{Mi}}(t)\right|} \text {. }
$$

Ness et al. (1971) suggested the use of Eq. (6) to determine if the spacecraft field changes during a time interval $d t$. On the assumption that the spacecraft magnetic field attenuates with increasing distance from the spacecraft and neglecting instrument noise, this quantity will take the value of 1 when the spacecraft field doesn't change, otherwise it will lie between 0 and 1. Taking into account instrument noise, Eq. (6) will in reality never be exactly 1 when the spacecraft field doesn't change. Thus an error margin $e$ needs to be assigned, where $e$ is close to, but less than 1 . The amount that $e$ differs from 1 will depend on the noise levels for a particular instrument. To indicate when the spacecraft field changes over an interval $d t$, Eq. (6) can thus be re-written as the inequality in Eq. (7).

$$
\frac{\left|\boldsymbol{B}_{\mathrm{Mo}}(t+d t)-\boldsymbol{B}_{\mathrm{Mo}}(t)\right|}{\left|\boldsymbol{B}_{\mathrm{Mi}}(t+d t)-\boldsymbol{B}_{\mathrm{Mi}}(t)\right|}<e .
$$

However, Eq. (7) is also inherently a function of the natural field. Instead it seems more prudent to use changes of the quantity in Eq. (5) to determine when there is a change in the spacecraft field, since the magnitude of any change in this quantity is not a function of the natural field. The result in Eq. (5) is particularly useful if events in the spacecraft field (i.e. any discrete change in the spacecraft field which can be associated with a change in configuration of the spacecraft's systems) are to be categorised using characteristics such as their change in field vector.

A number of different spacecraft and scientific instruments produce distinctive patterns in the MAG data. A four hour interval of $\boldsymbol{B}_{\mathrm{Dx}}$ measured by MAG on 14 July 2006 is displayed in Fig. 1. Two distinct kinds of patterns are illustrated by Fig. 1. The most visible are abrupt changes of 40-60 nT. Eight such events can be seen in Fig. 1. These are caused by a change in angle of the solar array drive motors (SADM's). In addition 32 higher frequency changes with a smaller magnitude $(\sim 5 \mathrm{nT})$ are present in Fig. 1. These effects take place as pairs, one in the positive and one in the negative direction and which occur periodically throughout MAG data. Similar effects are observed concurrently in the other two vector components. However, these changes, which can be as small as several $\mathrm{nT}$ in magnitude, are superimposed onto a changing background magnetic field of several thousand $\mathrm{nT}$ in the differenced data sets. Figure 2 displays a full $24 \mathrm{~h}$ orbit of the differenced data which graphically shows this large background field, resulting from the domination of the data set by the inboard magnetometer measurement.

While it can be seen that visual identification of these dynamic events appears simple, non-autonomous manual 


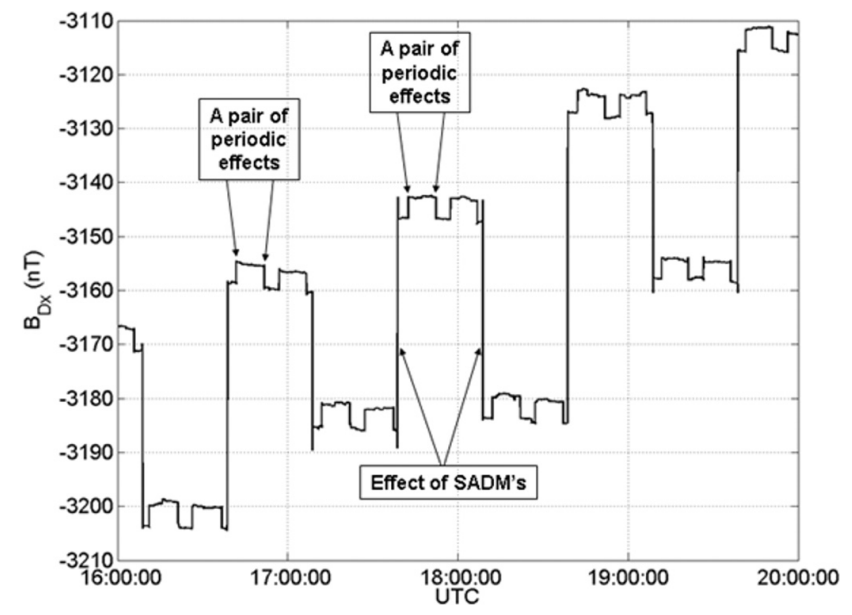

Fig. 1. $\boldsymbol{B}_{\mathrm{Dx}}(t)$ (spacecraft coordinate system) for $4 \mathrm{~h}$ measured on 14 July 2006. The data shows 8 effects ( 2 of these are illustrated) due to a change in angle of the solar array drive motors. 15 complete pairs of periodic spacecraft effects ( 2 are illustrated) are also present.

removal of spacecraft interference for the hundreds of significant events which occur every day is not a reasonable solution. Additionally, determining the contribution that each dynamic event makes to the two measured data sets is not trivial. The contribution from each event depends on the location, orientation, type and operational mode of its source. The problem is further complicated by the large spacecraft generated background field and the variable natural field, onto which these dynamic events are superimposed. The background spacecraft field can be three orders of magnitude greater than the events which need to be detected and the changes in the natural field can often be of a similar, or greater order as the dynamic events over a similar time interval. The resulting problem is to develop a methodology implemented in software that can automatically detect and remove the spacecraft generated magnetic field and instrument offset from the measured data.

The methodology should be able to achieve the following goals:

1. Use $\boldsymbol{B}_{\mathrm{D}}(t)$ to identify dynamic events in the spacecraft field, such as those illustrated in Fig. 1.

2. Determine the effect that these dynamic spacecraft events have on the measured field and correct the data to minimize these.

3. Calculate and remove the constant offset remaining in the measured values (sum of any remaining constant spacecraft field and sensor offset) to produce a data set containing solely the natural field.

If careful consideration is paid to the methods used to correct for the dynamic fields, the static spacecraft field will remain
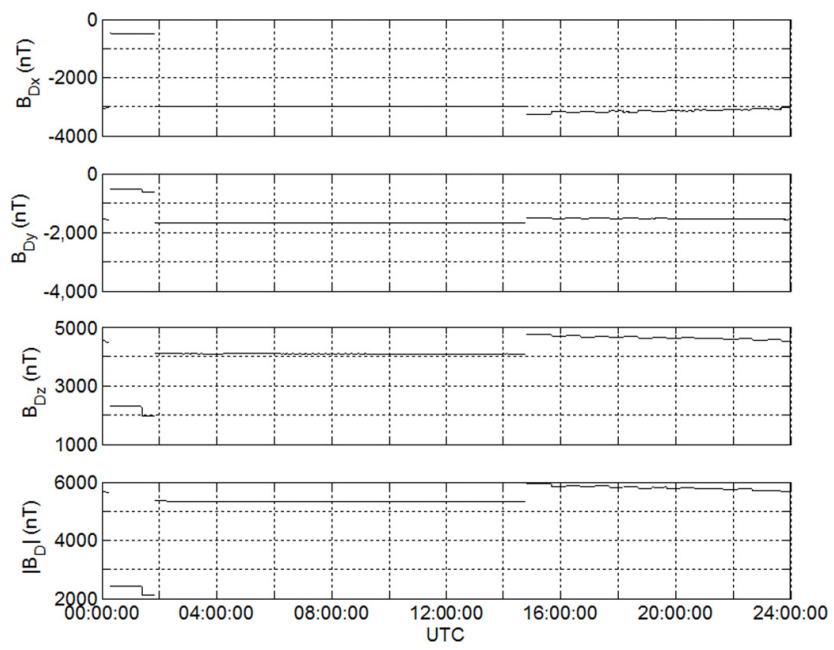

Fig. 2. $\boldsymbol{B}_{\mathrm{D}}(t)$ for the orbit $(24 \mathrm{~h})$ on 14 July 2006 in the spacecraft coordinate system.

approximately constant throughout each data set. Combining the static spacecraft contribution with the instrument offset creates a single offset. This can be determined using a modified Davis-Smith method (Leinweber et al., 2008), once the dynamic spacecraft contributions have been removed.

The magnitude of the spacecraft field $\left|\boldsymbol{B}_{\mathrm{S}}\right|$ in MAG data is relatively large compared to $\left|\boldsymbol{B}_{\mathrm{N}}\right|$. However, the fall off in total magnitude of the measured field from the inboard to outboard sensor is substantial. The ratio $\left|\boldsymbol{B}_{\mathrm{Mi}}\right| /\left|\boldsymbol{B}_{\mathrm{Mo}}\right|$ is typically greater than 10 , indicating that $\boldsymbol{B}_{\mathrm{Co}}$ is considerably smaller than $\boldsymbol{B}_{\mathrm{Ci}}$. This attenuation is such that a significant amount of the dynamics of the spacecraft field measured at the outboard sensor, are negligible. In this context, negligible is defined as a maximum field change of less than $0.1 \mathrm{nT}$ over the duration of a dynamic event measured at the outboard sensor. Thus the set of spacecraft disturbances which need to be removed to attain values of $\boldsymbol{B}_{\mathrm{N}}$ that are sufficiently free of dynamic interference, is significantly smaller for the outboard sensor in comparison with the inboard sensor. However, in spite of this reduction, the number of dynamic spacecraft contributions registered by the outboard sensor is still high.

\section{Detection of dynamic interference in MAG data}

The spacecraft magnetic field has a very complex nature due to the variety of different types and patterns of spacecraft interference. This signal is too complex to be dealt with in the framework of the methods proposed by Ness et al. (1971), due to their reliance on specific assumptions and approximations made about the spacecraft field, instrument design and properties of the natural field. Preliminary investigations also ruled out a completely automated black box approach because of this complexity. An alternative approach has been 


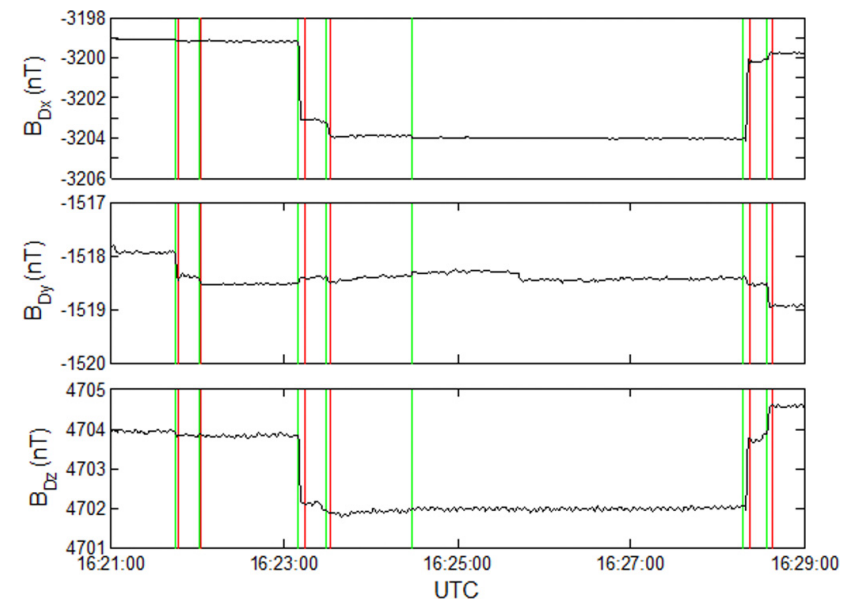

Fig. 3. Eight minutes of $\boldsymbol{B}_{\mathrm{Dx}}(t)$ measured on 14 July 2006 with the events (changes in the spacecraft magnetic field) detected by the fuzzy logic based algorithm marked. The green and red lines mark the start and end of these transient events, respectively.

adopted for MAG where patterns identified in the magnetic signature of VEX and information about the operation of the spacecraft systems have been adopted to separate different types of spacecraft interference. Using this information analytical and statistical modeling techniques have been used to correct the measured field. This requires prior information about the events to be collated and analyzed.

An autonomous method has been developed, specifically for VEX MAG, to detect the start and end of any response that contributes to the dynamic spacecraft interference. This algorithm serves two purposes; firstly it provides the timing and field information required for the "training" phase, where the different events are classified and modeled; secondly it allows a data set to be automatically divided into separate spacecraft dynamic disturbances required by point (1) in the actual cleaning process outlined in Sect. 2. This algorithm is very efficient in the automatic identification of the start and end of a wide range of spacecraft generated disturbances. It looks for either a bimodal distribution or a significant change in variance for a window of the time series of the $\boldsymbol{B}_{\mathrm{D}}$ field vector. A fuzzy logic based system is used to classify the distribution of the data by mapping it to a set of membership functions. The data is pre-processed to the range [0 1] prior to the application of the fuzzy logic detection algorithm. It is mapped to the range [ $\left[\begin{array}{ll}0 & 1\end{array}\right]$ so that a fixed set of membership functions can be used. The map has been specifically designed to preserve the required characteristics in the data. The bimodal distribution is typical for events in the spacecraft field which have a notable change in the static field over their complete duration. It is also more pronounced when the duration of the event is short compared to the length of the window. The algorithm also monitors the variance of the data to increase reliability and to ensure that all types of events, including those with minimal steady state change (i.e. the
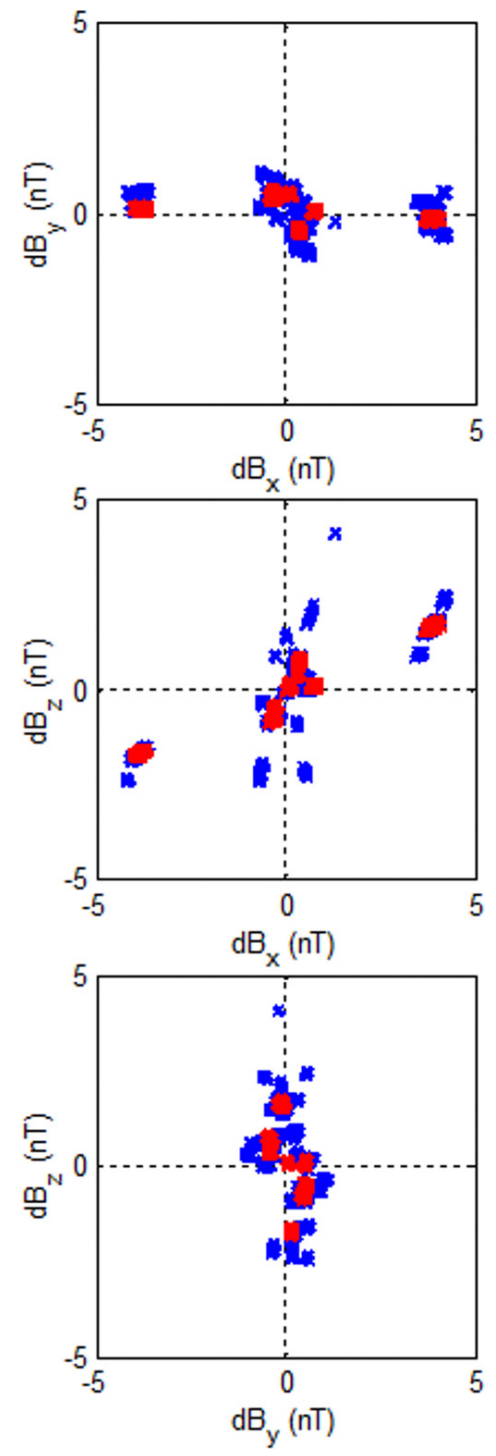

Fig. 4. 2-dimensional projections of the clusters identified from the full 3 components of $\boldsymbol{B}_{D}(t)$. The red and blue points mark the primary and remaining clusters, respectively.

change in field observed between the start and end points of any discrete transient event), are identified.

Figure 3 provides an example of the results attained by running the algorithm over a period of VEX MAG differenced data. The green and red lines mark the start and stop points, respectively, of the detected transient events. The algorithm is able to detect events in $\boldsymbol{B}_{D}$ with a very high degree of certainty, which have a steady state change in a single vector component greater than $1 \mathrm{nT}$. The algorithm is also capable of detecting much smaller events, just above the noise threshold for the measurements. The full details of this algorithm are beyond the scope of this paper and will be discussed in detail in a subsequent paper. 


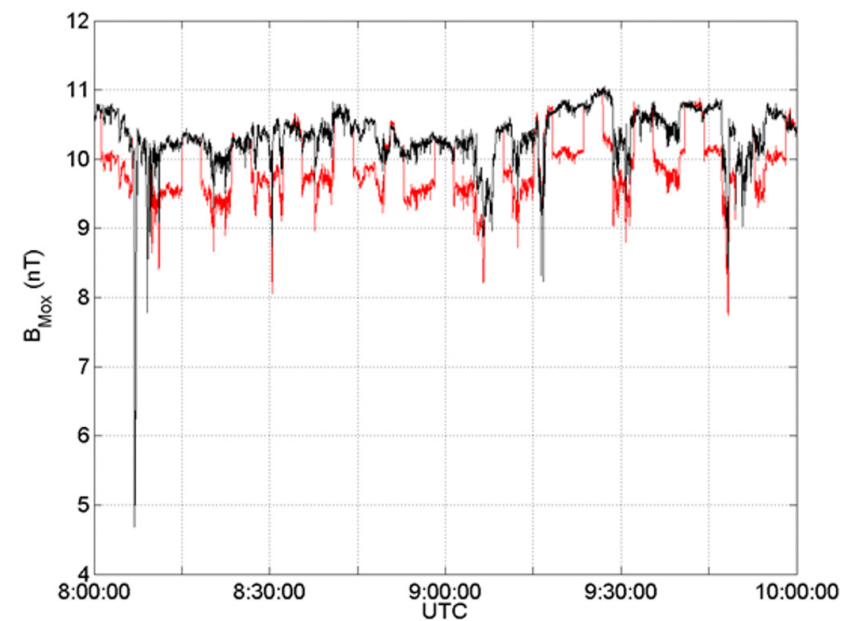

Fig. 5. A comparison of $2 \mathrm{~h}$ of outboard sensor data from the orbit on 9 June 2006. The data is in the spacecraft co-ordinate system and has offset corrected. Red: data before correction, black: data after correction for 2 types of periodic interference.

\section{Identification and correction of dynamic interference in MAG data}

Typically more than 1500 events per day are detected in MAG data by the previously described algorithm. Out of these, several hundred make a significant contribution to $\boldsymbol{B}_{\text {Mo }}$. Analysis of the events in $\boldsymbol{B}_{\text {Mo }}$ indicates that there are several types of spacecraft generated interference which comprise the majority of these events. Using a combination of VEX housekeeping data and pattern identification algorithms correlated with MAG measurements, most of these events can be attributed to the following categories:

- Periodic changes

- Antenna effects

- SADM (Solar Array Drive Motor) angle changes

- VIRTIS (Visible and Infra-Red Thermal Imaging Spectrometer) effects

- ASPERA (Analyser of Space Plasmas and Energetic Atoms) effects

- Reaction Wheel effects

- Slow/Thermal drift

The task of distinguishing individual events is not a straightforward one. The reason for this is that a detected response may be the result of a single individual event, or the sum of multiple systems operating in parallel. This can lead to a large variety of dynamics, which can range from a simple step response to those of a very complex nature and appearance.

\subsection{Periodic and antenna interference}

The periodic effects occur as pairs (this is illustrated in Fig. 1) and at the rate of at least 50 every $24 \mathrm{~h}$. The statistical properties of these events have been analyzed and several different types of pairs have been identified. Figure 4 displays the clusters identified in the differenced data and projected into the three two-dimensional measurement planes. The data plotted is the steady state change in the detected events. The clusters which have a significant effect in the outboard sensor measurements are marked as the red clusters. The pairs are clearly identified as the clusters with equal and opposite vector components. These clusters have been identified using a newly defined agglomerative clustering algorithm which uses a proximity measure similar to the $L^{1}$ Norm, but is designed such that an initial clustering result replaces the need for a termination threshold on the proximity measure. The details of this algorithm are the subject of a manuscript currently under preparation for publication in a suitable subject specific journal. Optimal corrections for these identified pairs at both sensor locations can be determined from their statistical properties, with these giving a $0.69 \mathrm{nT}$ correction for the largest pair in $\left|\boldsymbol{B}_{\mathrm{Mo}}\right|$. A specifically designed pattern recognition algorithm together with these correction factors and the record of events from the detection algorithm previously described are used to identify and correct associated pairs. By only correcting pairs the static offset is not influenced. Figure 5 displays $2 \mathrm{~h}$ of $\boldsymbol{B}_{\text {Mox }}$ from 9 June 2006 illustrating the result of correcting for the two types of most significant periodic effects. The nominal correction method is to remove the data during the transient phase. This is the most suitable approach since they occur as almost step like changes, leading to minimal data removal. The specific sources of these events have not been identified in the operation of the spacecraft and its subsystems and there is no need to identify their source. A similar method can be applied to the antenna effects, the only difference being that these events occur more irregularly, but with a much bigger contribution in the measured field vectors. These events have a maximum contribution in $\left|\boldsymbol{B}_{\text {Mo }}\right|$ of $56.1 \mathrm{nT}$.

\subsection{SADM interference}

The results from the initial detection algorithm are correlated with the time and angle changes for the SADM's identified using housekeeping data. Statistically accurate models, relating SADM angle to magnetic field contribution at the two sensor locations, can be derived from this dataset. Two models have been trained, one for the differenced and one for the inboard data set. These two data sets have the greatest ratio of SADM field to local natural field, i.e. signal to noise ratio, where the noise in this case is the local natural field. The model for the SADM's measured at the outboard sensor is calculated as the difference of these two models. The 


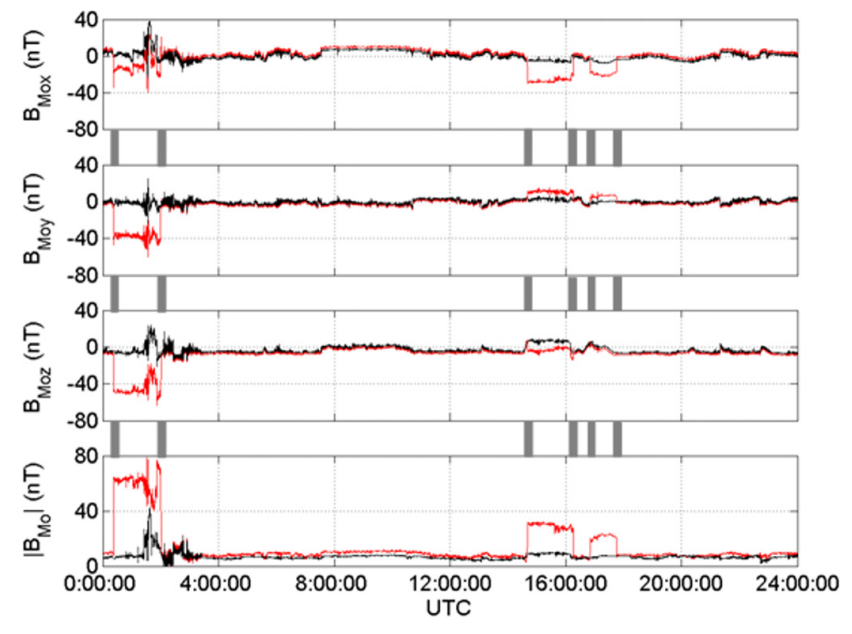

Fig. 6. A comparison of $24 \mathrm{~h}$ of outboard sensor data from the orbit on 9 June 2006. The data is in the spacecraft co-ordinate system and with offset corrected. Red: data before correction, black: data after correction for SADM, Antenna and Periodic effects. The grey lines mark times when interference from the SADM occurs in the original data.

effect of the SADM's in the measured datasets is corrected using a combination of the general detection results, housekeeping data and the model. The knowledge of the angle change elicited from the low time resolution housekeeping data is translated into an expected field change in $\boldsymbol{B}_{\mathrm{D}}$. This field change is used to identify the corresponding event in the magnetic field time series from the list of events in the time region defined by the low resolution housekeeping data. Currently the data is only corrected for the steady state change associated with each event. The data measured during each event is removed. Whilst this is a sub-optimal approach in terms of the amount of data removed, it does ensure that the cleaned data is free from spacecraft interference. Initial work on modeling the transient field for the SADM's has been successful and the final cleaning algorithms will in due course be modified to correct the transients and steady state field. Figure 6 shows $24 \mathrm{~h}$ of $\left|\boldsymbol{B}_{\mathrm{Mo}}\right|$ data both before and after correction for SADM, periodic, antenna and VIRTIS effects. The large events marked by the grey lines at approximately 00:30:00 and 02:30:00 UTC are both due to changes in SADM angle and the result of correcting these is clearly seen in the data. Further development of this correction procedure is currently being investigated, with the aim that it can become independent of the house keeping data once a model has been trained. This is achieved through the use of pattern recognition and non-linear optimization techniques, together with the knowledge that the starting angle of any change in SADM position is the same as the end angle of the previous SADM position change.
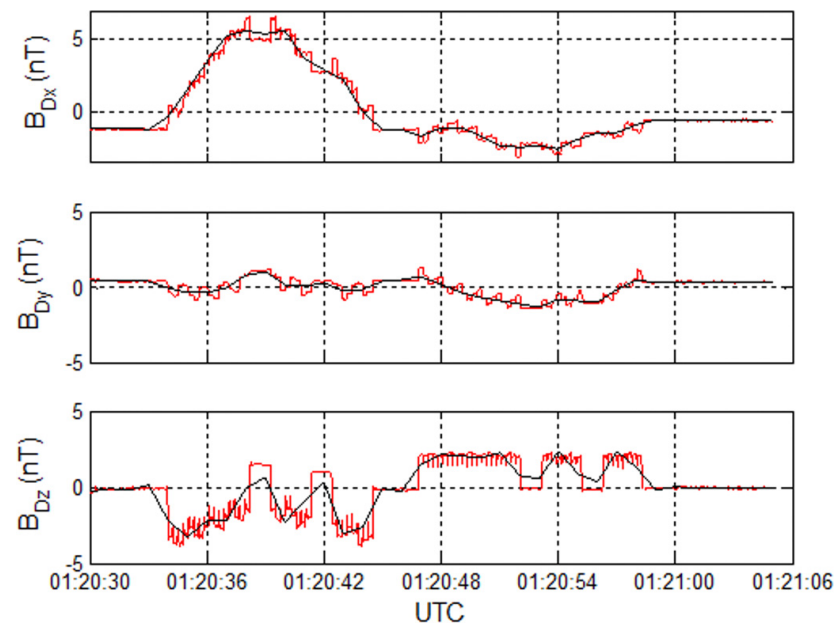

Fig. 7. A comparison of a VIRTIS associated event measured at $1 \mathrm{~Hz}$ (black) and $32 \mathrm{~Hz}$ (red) in the differenced data. The mean value of each field component has been removed to allow the relative dynamic contribution in the two data sets to be compared.

\subsection{VIRTIS interference}

The events associated with the VIRTIS experiment occur as purely transient events with duration in the region of $30 \mathrm{~s}$ and no significant change in steady state field. Following the detection of numerous events in the magnetic field measurements, their source was identified as a particular mode of the VIRTIS instruments through correlation with the housekeeping data. Importantly, knowledge of the source of these events isn't required to remove their effect from the measured field. The events have a spectral characteristic which has a well defined shape and is distinct from other events. This is more evident in higher frequency measurements, but there are significant components below $1 \mathrm{~Hz}$. Figure 7 displays a typical signal associated with the VIRTIS signal measured at both $1 \mathrm{~Hz}$ (black) and $32 \mathrm{~Hz}$ (red) in the differenced data. A spectral model normalized to the total power in the signal has been found to be sufficiently reliably to detect all of these types of event. However, it is not possible to reliably determine the time series of the signal in the measured field, once it has been filtered down to $1 \mathrm{~Hz}$. As a consequence the nominal correction procedure is to remove the section of data in the measured field contaminated by these events. This is acceptable since they have no influence on the steady state field. Despite their relatively long duration the impact of removing this data is small since their rate of occurrence is sufficiently low. For comparison, the total amount of data removed due to correction of these events is $5 \%$ of that removed for the most common type of the pair events.

\subsection{ASPERA interference}

Like with the VIRTIS events, the events associated with the ASPERA instrument are purely transient in nature. The 

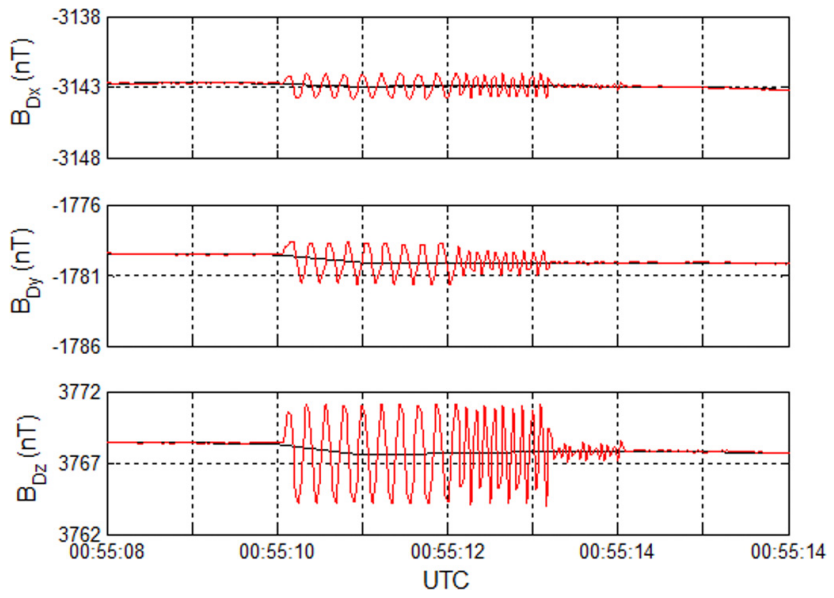

Fig. 8. Comparison of the ASPERA event measured at $1 \mathrm{~Hz}$ (black) and $32 \mathrm{~Hz}$ (red) in the differenced MAG data.

detected event is associated with a particular mode switch of the instrument, which occurs several times every $24 \mathrm{~h}$ orbit. The event is an oscillatory signal with approximately zero mean and a frequency which is observed to change in almost discrete steps in the high sample rate data. Figure 8 shows an APSERA event measured at both $1 \mathrm{~Hz}$ (black) and $32 \mathrm{~Hz}$ (red) in the differenced data. The event is well defined in the $32 \mathrm{~Hz}$ data, but not present in the $1 \mathrm{~Hz}$ data. This arises from the combined effect of the signals frequency, which is greater than the Nyquist frequency of the $1 \mathrm{~Hz}$ data and the averaging effect of the filter used to down-sample the data. This is the case for all of the $1 \mathrm{~Hz}$ data and a statistical analysis leads to the conclusion that there is no significant steady state change. As a result no action is required to compensate for the effect from the APSERA instrument in the $1 \mathrm{~Hz}$ data.

\subsection{Reaction wheel interference}

Reaction wheels are well known as a cause of interference in the magnetic field measurements made by spacecraft (e.g. Narvaez, 2004; Glassmeier et al., 2007). The angular velocities of the reaction wheels on Venus Express change in discrete intervals several times over the course of each $24 \mathrm{~h}$ orbit. Their operation leads to an oscillatory signal with approximately zero mean and a frequency which is observed to vary slowly in the high sample rate data. Figure 9 shows a reaction wheel event measured at both $1 \mathrm{~Hz}$ (black) and $32 \mathrm{~Hz}$ (red) in the outboard data. For the same reasons as outlined for the APSERA events, it is well defined in the $32 \mathrm{~Hz}$ data, but not present in the $1 \mathrm{~Hz}$ data and as a result no action is required to compensate for the effect of the reaction wheels in the $1 \mathrm{~Hz}$ data.
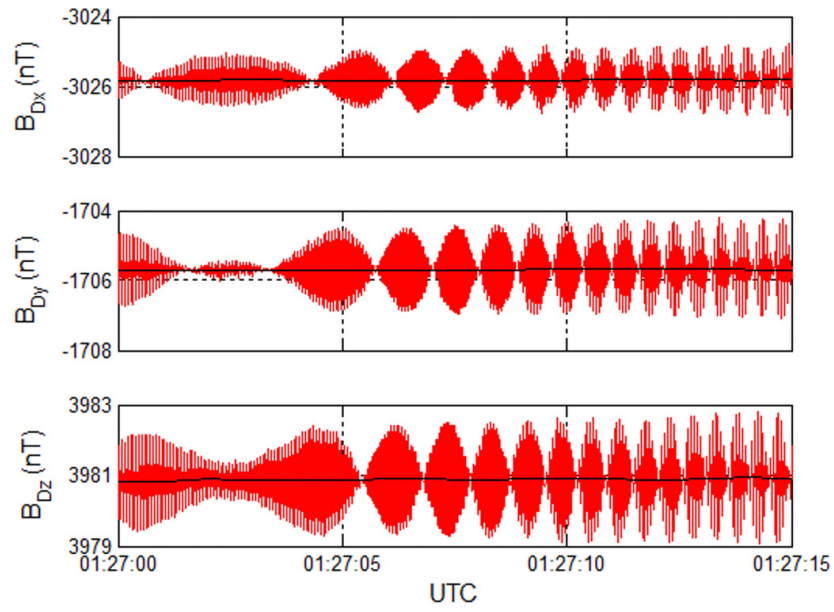

Fig. 9. Comparison of a Reaction Wheel event measured at $1 \mathrm{~Hz}$ (black) and $32 \mathrm{~Hz}$ (red) in the differenced MAG data.

\subsection{Slow/thermal drift}

The differenced data contains a slow drift of several nT which appears to be approximately periodic over each orbit. It has several possible causes including internal heating and solar radiation effects on the spacecraft, which are modulated by its orbit and attitude. The effect has not been observed in $\boldsymbol{B}_{\mathrm{Mo}}$, most likely due to a substantial attenuation at the outboard sensor. Thus, no steps have been taken to correct $\boldsymbol{B}_{\text {Mo }}$ for this effect.

\subsection{Discussion of the identification and cleaning results}

Figure 6 shows one orbit ( $24 \mathrm{~h}$ ) of MAG data in the spacecraft coordinate system. Two data sets are shown and both of these have been corrected for the combined offset determined through the technique outlined in Leinweber et al. (2008). The data shown in red is before any type of dynamic spacecraft field has been corrected, i.e. $\boldsymbol{B}_{\mathrm{N}}+\boldsymbol{B}_{\mathrm{Sd}}$. The data shown in black is after correction for SADM, antenna, periodic and VIRTIS effects, i.e. estimated $\boldsymbol{B}_{\mathrm{N}}$. It is these four types of dynamic spacecraft interference which comprise most of the interference in $\boldsymbol{B}_{\mathrm{Mo}}$. The two events at approximately 14:40:00 and 16:15:00 UTC are the result of a rotation of the spacecraft combined with an almost simultaneous realignment of the solar panels. The data is shown in the spacecraft co-ordinate system. This is why the corrected field appears different in the $\boldsymbol{B}_{\mathrm{Nz}}$ component and the total field $\left|\boldsymbol{B}_{N}\right|$ remains approximately constant, as expected when a spacecraft rotates in the solar wind. For comparison, Fig. 10 displays the same data as Fig. 6, but in the Venus-Sun-Orbit coordinate system. In this co-ordinate system, which is fixed in reference to the orbit of Venus, the aforementioned effect due to the rotation of the spacecraft is removed and the measured field does not deviate significantly over this short time period. The data plotted in Fig. 10, constitutes the final cleaned 

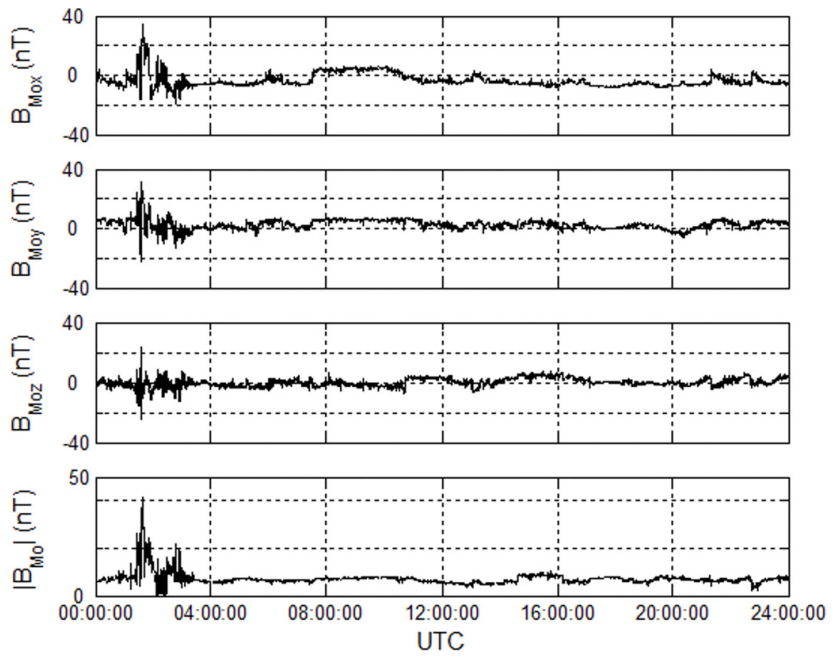

Fig. 10. $24 \mathrm{~h}$ of $1 \mathrm{~Hz}$ VEX MAG science data from the orbit on 9 June 2006 and plotted in the Venus-Sun-Orbit co-ordinate system.

data which is used for scientific analysis. These examples illustrate the power of the developed methodology to identify and correct spacecraft generated interference in the measured field. Any remaining dynamic interference is minimal and it is more convenient to remove this manually following the automated correction procedures. The result is a data set that is estimated to have a dynamic field accuracy of much better than $0.1 \mathrm{nT}$ and is comparable to the measurements made from onboard a magnetically clean spacecraft (Zhang et al., 2008a, b). The statement that the data is of comparable quality to measurements made from onboard magnetically clean spacecraft, is also supported by the comparison of magnetic field data during the period when Venus Express and MESSENGER were both in the solar wind in close proximity to Venus (Slavin et al., 2009).

The post measurement cleaned magnetic field data has provided many interesting results throughout the science phase of the Venus Express mission. These endorse the measurement philosophy which has been adopted for the first time with Venus Express. Such studies include Zhang et al. (2007), in which the interaction of the solar wind with Venus during solar minimum was studied. Previous investigations of this interaction had been conducted at solar maximum. This study concluded that at solar minimum the measurements made by VEX are consistent with a complete deflection of the solar wind around the ionosphere of Venus, i.e. minimal plasma from the solar wind enters the atmosphere of Venus. The loss of matter from the atmosphere and its affect on the evolution of the atmosphere of Venus is one of the important questions high-lighted for Venus Express. Zhang et al. (2007) suggest that the curved magnetic field configuration in the night-side ionosphere and tail close to the planet could lead to acceleration of plasma, resulting in some atmospheric loss. Pope et al. (2009) have detected what appear to be large wave like structures on the boundary between the ionosphere and magnetic barrier. These waves develop into structures which indicate a vortex configuration, which has previously been linked to the loss of atmospheric plasma (Wolff et al., 1980). These structures could also aid in the redistribution of ionospheric plasma created in the day-side through to the night-side during the long (58 terrestrial day) nights. Delva et al. (2008) studied proton cyclotron waves in the solar wind upstream of Venus. The occurrence of these waves near Venus suggests neutral planetary hydrogen atoms extend away from Venus. These atoms are ionised through various processes, picked-up by the solar wind in the form of protons and transported away from the planet.

Zhang et al. (2008b) used VEX MAG data to study the location and structure of the magnetic barrier at Venus. The magnetic barrier plays the same role as the magnetosphere at planets with a significant intrinsic magnetic field, in that it provides the effective barrier to the solar wind. The study shows that there is a well defined upper boundary to the magnetic barrier which can be considered as an induced magnetopause. Zhang et al. (2008a) used VEX MAG data to study the location of the Venusian bow shock at solar minimum. This study showed that the bow shock is located notably closer to Venus at solar minimum, when compared to studies conducted at solar maximum. For example, the solar minimum bow shock at the terminator is located at $2.14 R_{\mathrm{V}}$ (Venus Radii), compared to $2.40 R_{\mathrm{V}}$ at solar maximum. The bow shock also continues to remain completely detached at solar minimum. Further studies using VEX MAG data have discovered a new type of collisionless shock referred to as a kinematic subcritical shock (Balikhin et al., 2008). This shock crossing took place at an unusually distant location during a strong Interplanetary Coronal Mass Ejection (ICME) (Zhang et al., 2008c).

\section{Conclusion}

The Venus Express MAG experiment adopts a new philosophy in measuring the local magnetic field vector. As a consequence the measurements returned by the MAG experiment onboard Venus Express, contain a significant amount of spacecraft generated field effects. The use of simultaneous dual point measurements is crucial to separate the dynamic spacecraft effects from those of the natural field. Using computational analysis and modeling techniques, a set of algorithms have been developed which can accurately detect, identify and correct the measured field for the dynamic spacecraft effects. Importantly, knowledge of the source of the effect is often not needed for reliable detection and correction. The resulting cleaned datasets are of comparable quality to magnetic field measurements made onboard magnetically clean spacecraft. These cleaned data sets have been instrumental in studies which have discovered new plasma interactions while investigating the local plasma environment 
near Venus at solar minimum for the first time. These discoveries endorse the new magnetic field measurement approach which has been used for the first time with Venus Express.

Acknowledgements. The work in the UK was supported by the Science and Technology Facilities Council under grants ST/G004218/1 and ST/F002939/1. The work in China was supported by the National Science Foundation of China (NSFC) under grant 40931053.

Guest Editor M. Gedalin thanks two anonymous referees for their help in evaluating this paper.

\section{References}

Balikhin, M. A., Zhang, T. L., Gedalin, M., Ganushkina, N. Y., and Pope, S. A.: Venus Express observes a new type of shock with pure kinematic relaxation, Geophys. Res. Lett., 35, L01103, doi:10.1029/2007GL032495, 2008.

Carr, C., Brown, P., Zhang, T. L., Gloag, J., Horbury, T., Lucek, E., Magnes, W., O’Brien, H., Oddy, T., Auster, U., Austin, P., Aydogar, O., Balogh, A., Baumjohann, W., Beek, T., Eichelberger, H., Fornacon, K.-H., Georgescu, E., Glassmeier, K.-H., Ludlam, M., Nakamura, R., and Richter, I.: The Double Star magnetic field investigation: instrument design, performance and highlights of the first year's observations, Ann. Geophys., 23, 27132732, doi:10.5194/angeo-23-2713-2005, 2005.

Delva, M., Zhang, T. L., Volwerk, M., Voeroes, Z., and Pope, S. A.: Proton cyclotron waves in the solar wind at Venus, J. Geophys. Res.-Planets, 113, E00B06, doi:10.1029/2008JE003148, 2008.

Glassmeier, K.-H., Richter, I., Diedrich, A., Musmann, G., Auster, U., Motschmann, U., Balogh, A., Carr, C., Cupido, E., Coates, A., Rother, M., Schwingenschuh, K., Szegö, K., and Tsurutani, B.: RPC-MAG The Fluxgate Magnetometer in the ROSETTA Plasma Consortium, Space Sci. Rev., 128, 649-670, 2007.

Leinweber, H. K., Russell, C. T., Torkar, K., Zhang, T. L., and Angelopoulos, V.: An advanced approach to finding magnetometer zero levels in the interplanetary magnetic field, Measurement Sci. Technol., 19, 055104, doi:10.1088/0957-0233/19/5/055104, 2008.

Narvaez, P.: The Magnetostatic Cleanliness Program for the Cassini Spacecraft, Space Sci. Rev., 114, 385-394, doi:10.1007/s11214004-1433-1, 2004.

Ness, N. F., Behannon, K. W., Lepping, R. P., and Schatten, K. H.: Use of Two Magnetometers for Magnetic Field Measurements on a Spacecraft, J. Geophys. Res., 76, 3564-3573, 1971.

Ness, N. F., Behannon, K. W., Lepping, R. P., Whang, Y. C., and Schatten, K. H.: Magnetic field observations near Venus: Preliminary results from Mariner 10, Science, 183, 1301-1306, 1974.

Pope, S. A., Balikhin, M. A., Zhang, T. L., Fedorov, A. O., Gedalin, M., and Barabash, S.: Giant Vortices lead to Ion Escape from Venus and re-distribution of plasma in the Ionosphere, Geophys. Res. Lett., 36, L07202, doi:10.1029/2008GL036977, 2009.
Slavin, J. A., Acuna, M. H., Anderson, B. J., Barabash, S., Benna, M., Boardsen, S. A., Fraenz, M., Gloeckler, G., Gold, R. E., Ho, G. C., Korth, H., Krimigis, S. M., McNutt, R. L., Raines, J. M., Sarantos, M., Solomon, S. C., Zhang, T., and Zurbuchen, T. H.: MESSENGER and Venus Express observations of the solar wind interaction with Venus, Geophys. Res. Lett., 36, L09106, doi:10.1029/2009GL037876, 2009.

Snare, R. C. and Means, J. D.: A Magnetometer for the Pioneer Venus Orbiter, IEEE Transactions on Magnetics, 13, 1107-1109, 1977.

Wolff, R. S., Goldstein, B. E., and Yeates, C. M.: The onset and development of Kelvin-Helmholtz instability at the Venus ionopause, J. Geophys. Res., 85, 7697-7707, 1980.

Zhang, T. L., Baumjohann, W., Delva, M., Auster, H.-U., Balogh, A., Russell, C. T., Barabash, S., Balikhin, M., Berghofer, G., Biernat, H. K., Lammer, H., Lichtenegger, H., Magnes, W., Nakamura, R., Penz, T., Schwingenschuh, K., Vörös, Z., Zambelli, W., Fornacon, K.-H., Glassmeier, K.-H., Richter, I., Carr, C., Kudela, K., Shi, J. K., Zhao, H., Motschmann, U., and Lebreton, J.-P.: Magnetic field investigation of the Venus plasma environment: Expected new results from Venus Express, Planet. Space Sci., 54, 1336-1343, 2006.

Zhang, T. L., Delva, M., Baumjohann, W., Auster, H.-U., Carr, C., Russell, C. T., Barabash, S., Balikhin, M., Kudela, K., Berghofer, G., Biernat, H. K., Lammer, H., Lichtenegger, H., Magnes, W., Nakamura, R., Schwingenschuh, K., Volwerk, M., Vörös, Z., Zambelli, W., Fornacon, K.-H., Glassmeier, K.-H., Richter, I., Balogh, A., Schwarzl, H., Pope, S. A., Shi, K., Wang, C., Motschmann, U., and Lebreton, J.-P.: Little or no solar wind enters Venus' atmosphere at solar minimum, Nature, 450, 654-656, 2007.

Zhang, T. L., Delva, M., Baumjohann, W., Volwerk, M., Russell, C. T., Barabash, S., Balikhin, M., Pope, S. A., Glassmeier, K.-H, Kudela, K., Wang, C., Vörös, Z., and Zambelli, W.: Initial Venus Express magnetic field observations of the Venus bow shock location at solar minimum, Planet. Space Sci., 56, 785-789, 2008a.

Zhang, T. L., Delva, M., Baumjohann, W., Volwerk, M., Russell, C. T., Wei, H. Y., Wang, C., Balikhin, M., Barabash, S., Auster, H. U., and Kudela, K.: Induced magnetosphere and its outer boundary at Venus, J. Geophys. Res., 113, E00B20, doi:10.1029/2008JE003215, 2008b.

Zhang, T. L., Pope, S., Balikhin, M., Russell, C. T., Jian, L. K., Volwerk, M., Delva, M., Baumjohann, W., Wang, C., Cao, J. B., Gedalin, M., Glassmeier, K.-H., and Kudela, K.: Venus Express observations of an atypically distant bow shock during the passage of an interplanetary coronal mass ejection, J. Geophys. Res.-Planets, 113, E00B12, doi:10.1029/2008JE003128, 2008c. 